\title{
Amalgamation of Blockchain Technology and Internet of Things in Securing Clouds
}

\author{
Harsh Kumar Jha ${ }^{1}$, Durgesh Sahu' ${ }^{1}$, Dr. Mohammed Bakhtawar Ahmed ${ }^{2}$ \\ 'Student, Amity University Chhattisgarh, India \\ ${ }^{2}$ Faculty, Amity University Chhattisgarh, India
}

\begin{abstract}
Article Info

Volume 7, Issue 6

Page Number: 135-145

Publication Issue :

November-December-2021

\section{Article History}

Accepted : 20 Nov 2021

Published : 05 Dec 2021

Blockchain Technology has gotten a great deal of consideration from both industry and the scholarly world due to its decentralized, persistency, namelessness and auditability properties. In this review, utilization of Blockchain innovation in wide applications region and its execution challenges have been finished. A thorough quest for diary/research article identified with Blockchain innovation have been checked on. We have considered five data sets to lead this study specifically Science direct, IEEE Xplore, Web of Science, ACM Digital Library and Inderscience are being utilized. After starting stage end 135 examination articles are considered in last data sets for the overview. Principle focal point of the review is to give an extensive examination on wide utilizations of Blockchain innovation for the scholastic exploration local area. In this paper difficulties in carrying out of Blockchain and its related security and protection issues have been talked about. Interestingly a study of this sort have been done where Blockchain with application and its related security and protection issue have been inspected.

Keywords : Block Chain, IOT, Cloud Computing
\end{abstract}

\section{INTRODUCTION}

By utilizing encryption calculation, time stamp innovation, conveyed agreement and monetary motivators, blockchain innovation adequately tackles the agreement issue among associated hubs, and acknowledges decentralized shared exchange in a disseminated framework without common trust between hubs. Bitcoin framework is the most ordinary blockchain framework, and the construction of bitcoin-like blockchain framework is displayed in
Figure 1. In the blockchain framework, the digger hub utilizes the square header hash esteem as the pointers to interface the information hinders together to shape an unchangeable chain. Each square incorporates a square header and a square body. The square header epitomizes the metadata data of the current square, which gives the chance of following recorded exchange data. The square body fundamentally contains the exchange tree status data upheld by hash calculation. Every exchange is forever recorded in the information square, and anybody can 
question it. The Merkle tree in the square body will sign every exchange carefully, which can guarantee that each exchange is unforgeable and forestall twofold spend assault.

Blockchain innovation is improving and idiot proof security by eliminating go-betweens. It likewise brings about lessening the expense of exchanges. It is a common information structure that is manageable for gathering all the conditional history. In blockchain innovation, blocks are associated as chains. The starting square of the blockchain is perceived as the Genesis block. Any remaining squares are straightforward squares. The chain in the blockchain is the connection or the pointers interfacing the squares. Blocks, thus, keeps the exchanges that happen in the framework. Numerous associations have characterized Blockchain innovation in various ways. The Coinbase, the bulkiest digital money trade across the globe, has set up the blockchain as "An appropriated, public record that contains the historical backdrop of each bitcoin exchange" . Oxford word reference presents a natural definition expressed as "An advanced record in which exchanges made in bitcoin or another digital money are recorded sequentially and openly". One more portrayal is given by Sultan et al., which portrays an extremely broad meaning of blockchain innovation as "A decentralized data set containing consecutive, cryptographically connected squares of carefully marked resource exchanges, represented by an agreement model"

\section{COMPONENTS OF BLOCKCHAIN TECHNOLOGY}

Blockchain is an organization of squares (hubs) that are associated with each other after some geography rather than being associated with a focal server. It can possibly store the exchanges in the record viably and affirming straightforwardness, security, and auditability. Hardly any pivotal parts of blockchain innovation are as per the following.
1. Block: - Block in the blockchain innovation is the decentralized hubs/excavators furnished with the data sets, and it contains the advanced snippet of data. Blocks are connected together containing the hash worth of the past block into the current square. As a rule, block design can be envisioned into two sections: block header and a rundown of exchanges. Block header outfitted with the accompanying data:

- Version number shows the rendition number of the square and uses 4 bytes for its portrayal.

- Previous square hash is a pointer between the past and current square and uses 32 bytes.

- Timestamp utilizes 4 bytes and stores the hour of the production of the square. Merkle tree is addressed by 32 bytes and is a hash of each exchange that happens in a square.

- Difficulty target is demonstrated by 4 bytes and fundamentally it is utilized to quantify the unpredictability focus of the square.

- Nonce additionally utilizes 4 bytes and processes the various hashes

2. Beginning Block: - In a Blockchain, beginning square is considered as a foundered block since it is the main square in the chain. The Block stature of the primary square is consistently zero, and no square goes before the beginning square. Each square which is the piece of the blockchain involves a Block Header alongside Transaction Counter, and Transactions.

3. Nonce: - A nonce, an abbreviated version for "number just utilized once" is a one-time code in cryptography. Itis a number added to the hashed (scrambled) block in a blockchain. At the point when it is repeated, it guarantees the trouble level of enmity. The Nonce is the number for which blockchain excavators tackle an intricate issue. It is likewise connected with the timestamp to restrict its lifetime; that is the reason assuming one performs copy exchanges, and, after its all said and done an alternate Nonce is required.

4. Client and Miner: - A computationally progressed hub that attempts to take care of a mind-boggling issue (which requires high calculation power) to 
backtrack another square which is perceived as an excavator. The excavators are equipped for working alone or in an aggregate everyday practice to track down the answer for the given numerical issue. The method involved with finding a clever square is opened by dividing new exchange data between each client in the blockchain network. It is the obligation of every client to gather the new exchanges into squares and put their endeavours to track down the confirmation of-work of the square. Confirmation ofwork is characterized as a client is needed to tackle a computational complex riddle for distributing another square and the arrangement of the riddle will be its evidence. This entire peculiarity is known as verification of work.

5. Chain and Height: - In blockchain innovation, the chain is a virtual string that interfaces the excavators in the accrescent set of squares with hashes [11]. The chain continues to develop as and when another square is attached. Blocks in the chain are by and large demonstrated by their square tallness in the chain which is only an arrangement number beginning from nothing (0). The tallness of a square is characterized as the quantity of squares in the chain between the beginning square and the given square (for which stature is to be determined).

\section{Blockchain architecture}

Blockchain is the innovation behind Bitcoin. It is a public circulated information base which holds the encoded record. Blockchain is innovation in a worldwide data set that anybody, anyplace, with a web association, can utilize. In contrast to a conventional data set, which is possessed by focal party like banks and legislatures, a Blockchain doesn't have a place with anybody. With a whole organization taking care of it, bamboozling the framework by faking report, exchanges and other data become close to unimaginable. Blockchain store data forever across an organization among hubs. This isn't just decentralizing the data however disperses it as well. Every hub in the organization can store the neighbourhood duplicate of the Blockchain framework which is occasionally refreshed to have the consistency among all hubs. A Blockchain is a circulated calculation and data sharing stage which empower various hubs that don't confide in one another can take dynamic interaction. The issue in unified framework is weak link. In a decentralized framework there are various organize focuses which defeated the weak link. In an appropriated climate each hub altogether executes the work. In Fig. 1 shown fundamental engineering of Blockchain. Every client addressed as hub associated in a dispersed manner. Each hub kept a duplicate of the Blockchain list which is consistently refreshed. A hub can perform diverse movement like start an exchange, approve an exchange or perform mining.

\section{BLOCKCHAIN TECHNOLOGY IN IOT}

Blockchain innovation can assume a crucial part for different protection and security issues of the IoT. In IoT, detecting gadgets normally send the information at an incorporated area for handling purposes. Blockchain innovation replaces the focal server idea of IoT by presenting the idea of conveyed record for each exchange with genuine validation [10]. It guarantees that putting away the exchange subtleties with the mediators is as of now excessive on the grounds that exchange records will be accessible on numerous PCs of the chain. This framework dismisses the updating and penetrating of one PC. Nonetheless, to make it effective, multi-signature insurance is needed to approve an exchange. Assuming a programmer attempts to take the data by entering the organization, various copy duplicates are accessible on numerous PCs around the world. For hacking the blockchain network effectively, the agreement of over half of frameworks in the organization is required. 


\section{Blockchain Integration}

Combination of Blockchain with IoT opens another entryway and more extensive space of innovative work in the space of IoT applications [16, 20, 21]. Throughout the most recent couple of years, uncommon development in the field of IoT has been noticed, which empowers wide freedoms like access and portion of the data. Commonly, getting to and sharing data can instigate difficulties like security, protection, and trust among the imparting parties. Blockchain can settle different issues of IoT like protection, security, and dependability. The appropriated idea of blockchain innovation can dispense with single point disappointment and makes it solid. We as a whole mindful that blockchain has effectively demonstrated its significance in monetary exchanges with the assistance of cryptographic forms of money, like Bitcoin and Ethereum. It eliminated the outsider prerequisite between P2P instalment administrations. A couple of IoT empowering influences have picked the blockchain innovation and framed a consortium for normalization and solid incorporation of BIoT (Blockchain-IoT). It is a gathering of 17 organizations intended to empower security, versatility, heterogeneity, protection, and confidence in circulated structure with the assistance of blockchain innovation [16]. IoT gadgets can speak with each other either straightforwardly, gadget to gadget, or through blockchain innovation.

\section{Security in Blockchain and IoT}

The Internet of Things (IoT) is a construction of Machine-to-Machine (M2M) relationship, with no human association by any means. Henceforth setting up confidence with the interest of machines is an imposing test that IoT gear actually has not met extensively. The Blockchain (BC) can make strides as a medium in this cycle, for further developed adaptability, insurance of information, reliability, and security. This interaction should be possible by BC innovation to follow all gadgets which are associated with the IoT climate, and from that point onward, it is utilized to make conceivable and additionally synchronize all exchange handling. By utilizing the blockchain work, we can completely eliminate a Single Point of Failure (SPF) in IoT structure. In BC, information is scrambled utilizing different calculations like cryptographic calculations and hashing procedures. Hence, the capacity of BC gives further developed security administrations in an IoT. The capacity of $\mathrm{BC}$ innovation is to fix the computerized market. It has an assurance and holding both fundamental and primer worries of the capacity of the BC. The BC keeps the record of a gathering of successive and grouping of data exchanges since it tends to be perused as a gigantic arranged timestepping framework. The regulators are too worried in BC's ability to suggest secured, classified, quickly recognizable observing of exchanges. Along these lines, the BC can work with us to stay away from the altering and ridiculing of information by the association and getting the modern IoT gadgets [22]. The Blockchain (BC) records each exchange and gives a cross-line generally disseminated certainty. Ordinarily, it is conceivable that Trusted Third Party frameworks or focal area-based administrations can be vitiated or hacked. In $\mathrm{BC}$, when exchanges are affirmed by agreement then the square information are adequate to all. The BC can be developed as (1) permissioned network, which is for the most part a private organization, and (2) consent less, a public organization. Permissioned BC offers new protection and further developed admittance usefulness. The BC can resolve these sorts of difficulties easily, emphatically, and ably. It has been for the most part utilized for giving dependable and confirmed uniqueness enlistment, ownership track, and screen of items, supplies, and assets. IoT gadgets are not absolved, blockchain can personality all the associated IoT gadgets. 


\section{CONCLUSION}

In this work, this paper presents the foundation of the data set turn of events, various sorts of information bases and their benefits and hindrances, just as blockchain innovation. Then, at that point, this paper addresses three disseminated information bases: Hadoop DB, Hive, and Bigchain DB. At long last, the paper thinks about the presentation of embedding and questioning in these three information bases. In spite of the fact that blockchain innovation is as yet in its earliest stages, it will have incredible advancement potential later on. Bigchain DB has filled in the clear in the blockchain data set region, which depends on circulated engineering with the highlights of blockchain. Within a reasonable time-frame, blockchain and blockchain information base innovation will certainly thrive and serve the public economy and individuals' job. It very well may be utilized in numerous parts of social administration and coordinated into individuals' lives, like computerized money, credit contracts, personality the executives, protected innovation the board, etc.

\section{REFERENCES}

[1]. H. F. Atlam and G. B. Wills, "Technical aspects of blockchain and IoT," in Advances in Computers, vol. 115: Elsevier, 2019, pp. 1-39.

[2]. X. Li, P. Jiang, T. Chen, X. Luo, and Q. Wen, "A survey on the security of blockchain systems," Future Generation Computer Systems, 2017.

[3]. A. D. Dwivedi, G. Srivastava, S. Dhar, and R. Singh, "A decentralized privacy-preserving healthcare blockchain for IoT," Sensors, vol. 19, no. 2, p. 326, 2019.

[4]. K. Sultan, U. Ruhi, and R. Lakhani, "Conceptualizing blockchains: characteristics \& applications," arXiv preprint arXiv:1806.03693, 2018.

[5]. S. Nakamoto, "Bitcoin: A peer-to-peer electronic cash system," Manubot, 2019.
[6]. M. Nofer, P. Gomber, O. Hinz, and D. Schiereck, "Blockchain," Business \& Information Systems Engineering, vol. 59, no. 3, pp. 183-187, 2017.

[7]. K. Ashton, "That 'internet of things' thing," RFID journal, vol. 22, no. 7, pp. 97-114, 2009.

[8]. S. Kumar and Z. Raza, "Internet of Things: Possibilities and Challenges," in Fog Computing: Breakthroughs in Research and Practice: IGI Global, 2018, pp. 1-24.

[9]. T. Guarda et al., "Internet of Things challenges," in Information Systems and Technologies (CISTI), 2017 12th Iberian Conference on, 2017: IEEE, pp. 1-4.

[10]. N. M. Kumar and P. K. Mallick, "Blockchain technology for security issues and challenges in IoT," Procedia Computer Science, vol. 132, pp. 1815-1823, 2018.

[11]. L. Zhu, K. Gai, and M. Li, "Blockchain and Internet of Things," in Blockchain Technology in Internet of Things. Cham: Springer International Publishing, 2019, pp. 9-28.

[12]. M. S. Ali, M. Vecchio, M. Pincheira, K. Dolui, F. Antonelli, and M. H. Rehmani, "Applications of blockchains in the Internet of Things: A comprehensive survey," IEEE Communications Surveys \& Tutorials, vol. 21, no. 2, pp. 16761717, 2018.

[13]. A. Ghimire, "Brief Survey and Testbed Development for Blockchain Based Internet of Things," The University of Mississippi, 2020.

[14]. K. Delmolino, M. Arnett, A. Kosba, A. Miller, and E. Shi, "Step by step towards creating a safe smart contract: Lessons and insights from a cryptocurrency lab," in International conference on financial cryptography and data security, 2016: Springer, pp. 79-94.

[15]. F. Ahmad, Z. Ahmad, C. A. Kerrache, F. Kurugollu, A. Adnane, and E. Barka, "Blockchain in internet-of-things: architecture, applications and research directions," in 2019 International Conference on Computer and 
Information Sciences (ICCIS), 2019: IEEE, pp. 1- 6.

[16]. T. A. Syed, A. Alzahrani, S. Jan, M. S. Siddiqui, A. Nadeem, and T. Alghamdi, "A Comparative Analysis of Blockchain Architecture and its Applications: Problems and Recommendations," IEEE Access, vol. 7, pp. 176838-176869, 2019.

[17]. M. A. Khan and K. Salah, "IoT security: Review, blockchain solutions, and open challenges," Future Generation Computer Systems, vol. 82, pp. 395-411, 2018.

[18]. T. M. Fernández-Caramés and P. Fraga-Lamas, "A Review on the Use of Blockchain for the Internet of Things," IEEE Access, vol. 6, pp. 32979-33001, 2018.

[19]. N. Kshetri, "Blockchain's roles in strengthening cybersecurity and protecting privacy," Telecommunications policy, vol. 41, no. 10, pp. 1027-1038, 2017.

\section{Cite this article as :}

Harsh Kumar Jha, Durgesh Sahu, Dr. Mohammed Bakhtawar Ahmed, "Amalgamation of Blockchain Technology and Internet of Things in Securing Clouds", International Journal of Scientific Research in Computer Science, Engineering and Information Technology (IJSRCSEIT), ISSN : 2456-3307, Volume 7, Issue 6, pp.135-145, November-December-2021. Available at doi : https://doi.org/10.32628/CSEIT217624

Journal URL : https://ijsrcseit.com/CSEIT217624 